\title{
Physical activity, physical fitness and prevention: Role for the working population
}

\author{
Herbert Löllgen', Petra Zupet ${ }^{2}$, Norbert Bachl ${ }^{3}$ \\ I Practice for Sports and Cardiology, University of Mainz, Bermesgasse 32 b, D-42897 Remscheid \\ ${ }^{2}$ IMS Institute for Medicine and Sports, Cesta na poljane 24, 1000 Ljubljana, Slovenia \\ ${ }^{3}$ Em. Director of the Austrian, Center for Sports sciences and Director of the Dept. Sports \\ and Exercise Physiology, Auf der Schmelz 6, II50 Wien \\ herbert.loellgen@gmx.de
}

\begin{abstract}
Introduction: Physical inactivity and increasing daily screen time is an emerging health problem within the working population as well in the general population worldwide. This is called the exercise deficiency syndrom (EDS).On the other hand a large number of single studies as well of meta-analysis strongly support the positive health effects of regular physical activity. Discussion: Regular physical activity (PA) is now widely accepted as one of the most important factors to maintain or improve health and to prevent numerous non-communicable diseases. PA reduces risks of all-cause- and cardiovascular morbidity, mortality. Therefore, PA is a cornerstone in prevention and therapy of many diseases thus improving quality of life and longevity. PA also counteracts the effects of sitting time and sedentary lifestyle (EDS). PA acts like a drug: there are many indications, a non-linear doseresponse curve, many somatic and psychosomatic effects, few side effects and contraindications. Similar results can be observed for physical fitness assessed by maximal watt or Vo2 max in exercise testing. PA therefore is the real polypill for prevention and therapy of many diseases. Conclusion: There is now a general agreement and convincing evidence that regular PA including daily life activities are essentials for maintaining health in the working age population. For staying health and preserving health, everybody should reduce or avoid the four main risk factors: no smoking, regular PA, healthy diet and normal body weight.

Key words: Physical activity; exercise deficiency syndrom; fitness; exercise prescription; training
\end{abstract}


$\mathrm{R}$ egular physical activity and aerobic exercise training are related to a reduced risk of fatal and non-fatal coronary events in healthy individuals, subjects with coronary risk factors, and cardiac patients over a wide age range. A sedentary lifestyle is one of the major risk factors for CVD (Karmali et al., 2013). Physical activity and aerobic exercise training are therefore suggested by guidelines as a very important non-pharmacological tool for primary and secondary cardiovascular prevention (Piepoli et al., 2016, Löllgen et al., 2009). In the EU, $50 \%$ of the citizens are involved in regular aerobic leisure-time, and/or occupational physical activity and the observed increasing prevalence of obesity is associated with a sedentary lifestyle; moreover, probably less than one-third of patients eligible for cardiac rehabilitation are offered this service.

\section{Biological rationale}

Regular aerobic physical activity results in improved exercise performance, which depends on an increased ability to use oxygen to derive energy for work. Primary adaptations occur in the working muscles with increase of mitochondria and improved biochemical substances and enzymes thus improving local muscle endurance properties with increased oxygen extraction in the working muscle. This will be the basis for regular physical activity.

Moreover, myocardial perfusion can be improved by aerobic exercise, with an increase in the interior diameter of major coronary arteries, an augmentation of microcirculation, and an improvement of endothelial function. Additional reported effects of aerobic exercise are antithrombotic effects that can reduce the risk of coronary occlusion after disruption of a vulnerable plaque, such as increased plasma volume, reduced blood viscosity, decreased platelet aggregation, and enhanced thrombolytic ability and a reduction of arrhythmic risk by a favourable modulation of autonomic balance (Pescatello 2014, Piepoli et al., 2016, Rowe et al., 2014).

The preventive effects of regular activity also take place in older adults in a similar way as described above with a dependency on activity amount and intensity (Löllgen et al., 2009). Physical activity has positive effects on many of the established risk factors for CVD therefore reflecting a pleiotropic effect. Physical activity prevents or delayes the development of hypertension in normotensive subjects and reduces blood pressure in hypertensive patients (James et al., 2014). HDL cholesterol levels are increased, control of body weight is improved, and the risk of developing non-insulin-dependent diabetes mellitus is lowered by activity.

\section{Healthy subjects}

In healthy subjects, growing levels of both physical activity and cardiorespiratory fitness are associated with a significant reduction $(20-30 \%)$ in risk of all-cause and cardiovascular mortality, in anon-linear dose-response fashion (Löllgen et al., 2009, Shiroma et al., 2014) The evidence suggests that risk of dy- 
ing during a given period continues to decline with increasing levels of physical activity and cardiorespiratory fitness. This is true for both men and women and across a broad range of ages from childhood to the very elderly. These findings are derived from prospective non randomised cohort studies with predefined study groups, with physically active and a control group, a current standard in epidemiological research. Further evidence on the benefit of physical activity are derived from four large meta-analyses (Class I, Evidence A, Grade strong) (Löllgen, 2013, Löllgen et al., 2009) (Figure 1). These meta-analyses have been adjusted for confounding risk factors (i.e. smoking, diet etc.).

\section{Sedentary lifestyle}

In the last years, there is growing evidence that negative effects of sedentary lifestyle is significantly enhanced by sitting time per day, especially the socalled „screen-time”, that is watching TV, PC-work, PC games and surfing the internet. A recent meta-analysis confirmed this to be a significant cofactor for cardiovascular risks (Class I, Level A, Grade strong). Most of the mortality-reduction effect seems to rely on a decrease in cardiovascular and CHD mortality. The level of decreased coronary risk attributable to regular physical activity is similar to that of other lifestyle factors such as avoiding cigarette smoking or Mediterranean diet. The risk of CVD (including CHD and stroke) or CHD alone is significantly reduced in more physically active or fitter persons, with a relative risk reduction nearly twice as great for cardiorespiratory fitness (CFR) than for physical activity increase at all percentiles (Kokkinos et al., 2006, Kokkinos et al., 2016). A possible explanation for the stronger dose-response gradient for fitness than for physical activity is that fitness is measured objectively, whereas physical activity is assessed by self-reports and questionnaires that may lead to misclassification and bias towards finding weaker physical activity or health benefit associations (Moore et al., 2012). Altogether, sedentary life style, too much sitting and screen time over hours can be summarized as Exercise Deficiency Syndrom (EDS) as an important risk factor for many diseases in the working population.

\section{Physical activity intensity (Dose - response relationship)}

Some meta-analyses also present data on dose-response relationship for physical activity vs risk reduction. The results of all studies confirm a non-linear relationship (Shiroma et al., 2014). The most significant relative decrease of mortality occurs from sedentary lifestyle or inactive phase to low or moderate intensity of physical activity. This then means that little activity is better than nothing.

Change from moderate to vigorous activity increases training response and additionally reduces the relative risk for mortality, but to a lesser degree (percentage) than the first (moderate) intensity category. This is emphasized by the flattening of the dose response curve with very vigorous activity. Two re- 
cent single centred prospective cohort studies confirmed these results showing significant increased longevity in the physically active group by about 4 to 7 years. This can be converted to the reciprocal value indicating a mortality reduction with increased amount of activity of about 22 to $40 \%$ (Moore et al., 2012). These studies also underscore the non-linear relationship of mortality reduction with amount of physical activity (Class I, Level A, Grade strong).

\section{Training recommendation (FITT): Frequency, intensity, time, and type}

All health related medical and non-medical societies involved in healthy life style recommend regular physical activity for at least 150 min of moderate intensity on 3-5 days a week or at least $75 \mathrm{~min} /$ week for vigorous intensity for e.g. 3 days a week for healthy individuals (Class I, level A) (Figure 1). Longer duration and intensity of activities may further increase the health benefits with a lower extent only. Combination of both training modalities are possible. In general, intensity of exercise is superior to duration of exercise to improve physical capacity (Table 1). Volume in this context is frequency times duration and intensity. Available evidence suggests that the total weekly volume of physical activity/aerobic exercise training can be obtained by summing multiple daily bouts of exercise, each lasting $\geq 10 \mathrm{~min}$. Physical activity/aerobic exercise training should be distributed over most days of the week.

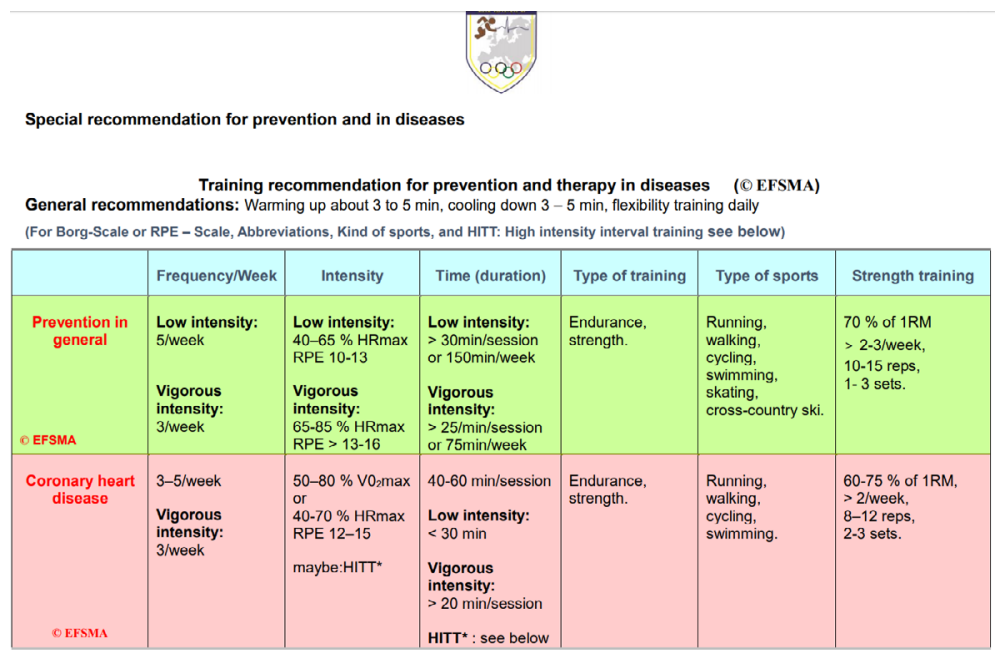

Figure 1: Examples for recommendations for physical activity prescription: Primary prevention and coronary heart disease (Zupet et al., 2015). 


\section{Types of activity}

Examples of physical activity/aerobic exercise training involve not only sport-related activities such as hiking, running or jogging, skating, cycling, rowing, swimming, cross-country skiing, and performing aerobic classes, but also lifestyle-common activities such as walking briskly, climbing stairs, and gardening work. In addition, engaging in active recreational pursuits contributes to health effects. Housework no longer has training effects due to the automatic machines used. In the elderly, walking and nordic walking is effective in promoting fitness.

\section{Prescription of exercise and physical activity: Frequency, intensity, time and type (FITT)}

In general, regular physical activity is prescribed like a drug (see below) by frequency, intensity, duration and type of activity (FITT). In addition, progression, and total amount are in use. Examples and criteria for exercise intensity are given in Table 1. For additional extensive fitness, adults may increase their aerobic activity up to 300 minutes a week of moderate-intensity, or 150 minutes a week of vigorous- intensity aerobic physical activity, thus increasing cardiopulmonary capacity. For long-term development and conservation of health related fitness and risk reduction, moderate intensity recommendations are first line and most important approach ( $150 \mathrm{~m} /$ week of moderate and $75 \mathrm{~min} /$ week of vigorous exercise). Ratings of perceived exertion related to exercise intensity with Borg scale are given in Table 1 . It can be easily used by healthy and ill subjects. The rate of progression within an exercise program depends on the individual's health and fitness status. To get started, the advice is start low and go slow. Details and comprehensive informations are given in the ACSM Guidelines (Pescatello et al., 2014) and Swedish recommendations (Swedish Nat. Inst. Public health, 2010). Strength training exercises are recommended to perform twice a week (Pescatello et al., 2014). A single exercise session includes warming-up, cooling down and flexibility exercises as part of preventive and rehabilitation program. For older adult at risk of falls, repeated exercises should be performed to maintain or improve balance and flexibility.

\section{Risk assessment}

To avoid complications and cardiac events during exercise, healthy subjects should be evaluated prior to engaging in regular physical activity/aerobic exercise training. The exercise-related risk of major cardiovascular events in ostensibly healthy people is exceedingly low, ranging from 1 in 500000 to 1 in 2600 ooo patient-hours of exercise.

As recently proposed for leisure-time sport activities in middle-aged/senior subjects, the risk assessment accuracy should be tailored to the individual's cardiac risk profile, the current level of habitual physical activity, and the intended level of physical activity/aerobic exercise training, with a more aggres- 
sive screening (i.e. exercise testing) for people who are sedentary, who are older (> 35ys), who start again with physical activity after long duration of inactivity and/or with cardiovascular risk factors.

Significant signs of latent diseases are dyspnoea, chest pain, palpitations, dizziness or even syncope. The current controversy on whether you can do too much of a good thing (i.e. exercise) should be kept in mind, but recent findings and many studies clearly show that the adverse effect of regular physical activity must be rejected due to many bias in the proposed studies (Sanchis-Gomar 2016). For the moment, there is a clear opinion that positive effects of regular exercise clearly outweigh the small risk, if any, in healthy subjects. Starting slow and go slow is the best advice to avoid a risk, especially in those subjects, which are adults or older adults and start again with exercise training. If maximal stress test is not feasible, a submaximal test such as 6-minutes walking test may be considered, although the correlation between $\mathrm{VO}_{2}$ peak and distance walked are moderate (Pescatello et al., 2014, Liu et al., 2014). Spiro-Ergometry, if available, is nowadays not only helpful but the gold standard for diagnosis of cardiorespiratory fitness and training counsellation.

\section{Exercise as a polypill}

Table 1: Evidence based indications for prescribing regular physical activity (Löllgen et al., 2013).

\begin{tabular}{ll}
\multicolumn{1}{c}{ Diseases } & Level of Evidence \\
Coronary artery disease & IA \\
\hline Artery Hypertension (4-8 mmHg) & IA \\
\hline Chronic obstructive lung disease & IA \\
\hline Heart failure & IA \\
\hline Cancer (colon, breast, lung) & IA \\
\hline Osteoporosis & IA \\
\hline Metabolic syndrome and Diabetes Mellitus & IA \\
\hline Chronic kidney disease & IA \\
\hline Peripheral arterial disease & IA \\
\hline Cognitive mental disorder & \\
$\quad$ Dementia & IB \\
\hline Mepression Alzheimer & IB \\
\hline Stroke & IB \\
\hline Fibromyalgie & IA \\
\hline Parkinson's disease & IA \\
\hline Chronic bowel disease & IB \\
\hline Bipolar disease & IA \\
\hline
\end{tabular}

Exercise prescription for health concept is similar to prescription of medication during drug therapy. It should also be applied for patients. Exercise as a drug depends on indications, dosage can be choosen individually, there is a (non-linear) dose-response relationship, regular exercise has somatic and psy- 
chosomatic effects, side effects are possible but rare, and contraindications are all acute diseases (Vina et al., 2012). Consequently, exercise prescription for health has now being introduced in many countries in Europe, North America and Australia /New Zealand. (Zupet et al., 2015; Vina et al., 2012; Löllgen et al., 2015; www.efsma.eu). It is unanimously recommended that regular aerobic exercise is encouraged in patients with heart failure and cardiovascular disease, esp. CAD, to improve functional capacity and symptoms (Class I, Level A, Grade: Strong).

\section{Preparticipation examination}

In patients, generally pre-participation examination is necessary, including stress testing and echocardiography if indicated. Spiroergometry as the golden standard improves informations on physical fitness. In patients with CVD, available data now allow definition of anaerobic exercise training weekly volume (frequency, intensity, time) similar to that indicated for healthy subjects (Table 3). This chapter and the following demonstrate the excellent positive effects of physical activities acting like a drug. Physical activity during rehabilitation after acute myocardial infarction reduces risk for death significantly by about 20 to $30 \%$. Risk for reinfarction does not change. Most studies confirm that physical training is the most important component of rehabilitation (Class I, level A). In a single randomised controlled trial, which enrolled 100 patients with single vessel coronary artery disease, intensive physical activity (daily training) had similar or better effect as PCI.

A meta-analysis including mainly middle-aged men, most of whom had a previous acute myocardial infarction and the rest with a previous CABG or percutaneous transluminal coronary angioplasty or affected by stable angina pectoris, showed a $30 \%$ reduction in total cardiovascular mortality for aerobic exercise training programmes of at least 3-months' duration. This percentage increased to $35 \%$ when only deaths from CHD were considered. Insufficient data were available on to the effects of aerobic exercise training on revascularization rates; moreover, aerobic exercise training did show no effect on the occurrence of non-fatal myocardial infarction.

In any case, recent data confirm the existence of an inverse dose-response relationship between cardiovascular fitness (evaluated by treadmill stress testing) and all-cause mortality in large populations of both male and female cardiovascular patients with a history of angiographically documented CHD, myocardial infarction, CABG, coronary angioplasty (PCI), chronic heart failure, peripheral vascular disease, or signs or symptoms suggestive of CHD during an exercise testing. The results were the same irrespective of use of beta-blocking agents. With moderate physical activity, incidence of cardiac arrhythmias may be reduced as has been shown in one single study. So, these findings are significant for working persons returning to work after cardiac events such as myocardial infarction. 


\section{Risk during training in cardiac diseases}

In general, the occurrence of major cardiovascular events during supervised aerobic training in cardiac rehabilitation programmes is rare: from 1 in 50 ooo to 1 in 120 ooo patient-hours of exercise, with fatality incidence ranging between 1 in 340 ooo and 1 in 750 ooo patient/hours of exercise. The same is also true for patients with chronic heart failure and reduced left ventricular function, New York Heart Association class II-IV symptoms, and treated with optimal, guideline-based background heart failure therapy. More evidences are needed therefore training supervision is now facilitated by tele monitoring.

\section{Heart Failure}

The effects of aerobic exercise training on cardiac mortality rate in patients with chronic heart failure have been evaluated in a meta-analysis. Overall, moderate to vigorous intensity aerobic exercise training resulted in improved survival in patients with chronic heart failure due to left ventricular systolic dysfunction, and time to readmission to hospital was also significantly extended. Regular physical activity not only reduces morbidity and rehospitalisation but also mortality. Left ventricular function increases as shown by Vo2max and ejection fraction. Physical capacity thus is improved and quality of life increased by 27 \% (Edelmann et al., 2011, Kitzmann et al., 2010).

Current multicenter study demonstrated that high intensity interval training (HIIT) in patients with cardiac failure can be safe and effective in increasing physical capacity (Class II a, Evidence B). During interval exercise, there are short bouts with high intensity (up to $90 \%$ of maximal exercise capacity) alternating with lower recovery periods. This kind of training improves cardiorespiratory fitness within short time (some months). HITT can also be combined with endurance training as a kind of basis training. However, one large, just finished multicenter study on HITT shows that there is no difference on the long time between endurance training or HIIT (Smartex-Project). Therefore, interval training will only be partly integrated into the physical activity program. Endurance training will be the mainstay of training in primary and secondary prevention. For competitive athletes, such a combination of longer duration endurance training together with longer intervals is well established since long.

\section{General recommendations for physical activity}

For long-term development and conservation of health related fitness and risk reduction, moderate intensity recommendations are first line and most important approach (150 $\mathrm{min} /$ week of moderate and $75 \mathrm{~min} /$ week of vigorous exercise). Ratings of perceived exertion related to exercise intensity are between 11 and 13, they can easily used by healthy and ill subjects. The rate of progression within an exercise program depends on the individual's health and fitness status. To get started, the advice is start low and go slow. Details and comprehen- 
sive informations are given in the inte website of EFSMA (www.efsma-scientific. eu), the ACSM Guidelines (Pescatello et al., 2014) and Swedish recommendations (Swedish Nat). On the EFSMA website, detailed recommendations for all diseases and for prevention are listed (Figure 1). Especially, intervention like physical activity improve work life- balance, prevent burn-out or depression and symptoms of fatigue thus enhancing satisfactory of working people.
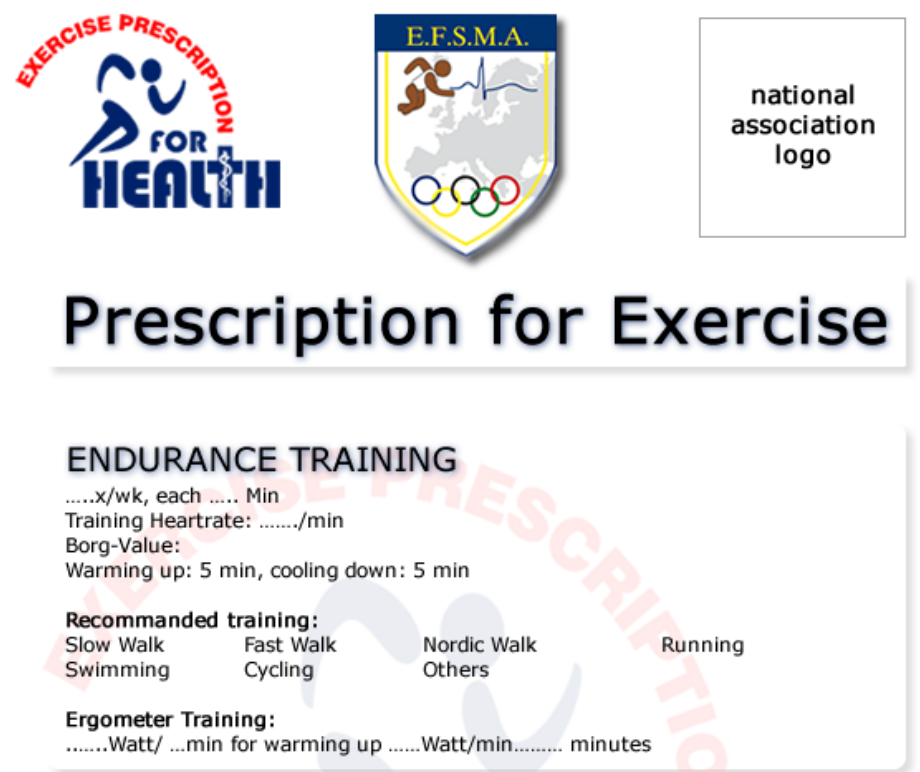

\section{STRENGTH TRAINING}

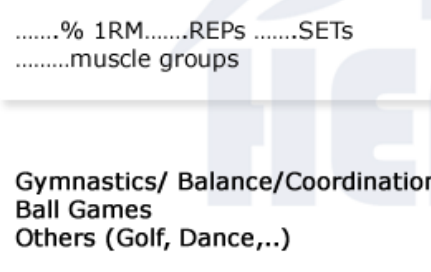

Sport Physician ....

In case of dyspnoe, irregular heart beats, chest pain or dizziness, stop activity and counsel your doctor.

Figure 2: Exercise Prescription for Health (Löllgen et al., 2013, Zupet et al., 2015).

Population based approaches to physical activities at the workplaces There is a need for comprehensive worksite fitness and wellness programmes together with education in healthy diet and nutrition. Fitness centers at work- 
site can be considered. Worksite programmes should be well structured to encourage working people to be active even during working time. Use of stairways should be encouraged with visible signs and short hints on the health promotion by using staircases instead of elevators. Repeated short time exercise bouts ( $5-10 \mathrm{~min}$. duration) during work can also be enough to reduce cardiovascular risk. The non-linear dose-response relationship between CFR and reduction in cardiovascular risk observed in primary prevention also applies to the secondary prevention setting. Advice that every doctor at every contact with a patient should ask for his physical activity improves patient's adherence to an active lifestyle. Role and sustainability of modern technology in improving health and motivating people to more physical activity. This comprises wearable technology, exergaming and App's to be downloaded.

\section{Conclusion}

Physical inactivity and sedentary lifestyle are among the three most frequent risk factors for cardiovascular diseases. Conversely, regular physical activity in prevention, rehabilitation and therapy, reduces cardiovascular morbidity, mortality and disability, improves cardiovascular function and quality of life as well as metabolic disorders. Similar effects are observed for hypertension, some central and peripheral diseases and diabetes mellitus. Effects of physical exercise are similar or superior to a single drug therapy. Exercise prescription for health should be used in healthy subjects and in all patients. Due to its pleiotropic action, physical activity has to be an essential part of prevention and therapy of in- and outpatient's therapy. Wearable technology will support training recommendations in the near future as do exergaming and many Aps which can be downloaded to the smartphone.

\section{References}

EDELMANN, F., GELBRICH, G., DÜNGEN, H.D., FRÖHLING, S., WACHTER, R., STAHRENBERG, R., et al., 2011. Exercise training improves exercise capacity and diastolic function in patients with heart failure and preserved ejection fraction: results of the Ex-DHF pilot study. J Am Coll Cardiol, vol. 58, pp. 1780-1791.

JAMES, P.A., OPARIL, S., CARTER, B.L., CUSHMAN, W.C., DENNISONHIMMELFARB, C., HANDLER, J., et al., 2014. Evidence - based guideline for the management of high blood pressure in adults. JAMA, vol. 311, pp. 507-520.

KITZMAN, D.W., BRUBAKER, P.H., MORGAN, T.M., STEWART, K.P., LITTLE, W.C., 2010. Exercise training in older patients with heart failure and preserved ejection fraction: a randomized, controlled single-blind trial. Circ Heart Fail, vol.3, pp. 657-667.

KARMALI, K.N., GOFF jr, D.C., NING, H., LLOYD-JONES, D.M., 2014. A systematic examination of the $2013 \mathrm{ACC} / \mathrm{AHA}$ pooled cohort risk assess- 
ment tool for atherosclerotic cardiovascular disease. J Am Coll Cardiol, vol.6o, pp. 959-968.

KOKKINOS, P.F., FASELIS, C., MYERS, J., NARAYAN, P., SUI, X., ZHANG, J., et al., 2016: Cardiorespiratory fitness and incidence of major adverse cardiovascular events in US veterans:Acohort study. Mayo Clin Proc.

KOKKINOS, P., PITTARAS, A., MANOLIS, A., PANAGIOTAKOS, D., NARAYAN, P., MANJOROS, D., et. Al., 2006. Eercise capacity and 24-h blood pressure in prehypertensive man and women. Am J Hypertension, vol. 19, pp. 251-258.

LIU, J., SUI, X., LAVIE, C.J., ZHOU, H., PARK, Y., CAI, B., LIU, J., BLAIR, S.N., 2014. Effects of cardiorespiratory fitness on blood pressure trajectory with aging in a cohort of healthy men. J Am Coll Cardiol, 2014, vol. 64, pp. 1245-53.

LÖLLGEN H., BÖCKENHOFF, A., KNAPP, G., 2009. Primary prevention by physical activity:An updated meta-analysis with different intensity categories. Int.J SportsMed, vol. 30, pp. 213-224.

LÖLLGEN, H., 2013. Bedeutung und Evidenz der körperlichen Aktivität zur Prävention und Therapie von Erkrankungen (Importance and evidence of regular physical activity for evention and treatment of diseases). Dtsch med.Wschr, vol. 138, pp. 2253-2259.

LÖLLGEN, H., BÖRJESSON, M., CUMMISKEY, J., BACHL, N., DEBRUYNE, A., 2015. The Pre-Participation Examination in Sports: EFSMA Statement on ECG for Pre-Participation Examination. Dtsch Zschr Sportmed, vol. 66, pp.151-155.

MOORE, S.C., PATEL, A.V., MATTHEWS, C.E., BERRINGTON DE GONZALEZ, A., YIKYUNG PARK, Y., KATKI, H.A., et al., 2012. Leisure Time Physical Activity of Moderate to Vigorous Intensity and Mortality: A Large Pooled Cohort Analysis. Plos one, vol. 9, e1001335.

PESCATELLO, L.S., (ED), 2014. ACSM's Guidelines for exercise testing and prescription $9^{\text {th }} \mathrm{ed}$, Wolters Kluwer, Baltimore.

PIEPOLI M.F., HOES, A.W., (Chairs), 2016. 2016 European guidelines on cardiovascular disease prevention in clinical practice guidelines. Europ Heart J, vol. 37, pp. 2315-2381.

ROWE, G.C., SAFDAR, A., ARANY, Z., 2014. Running Forward.New Frontiers in Endurance Exercise Biology. Circulation, vol. 129, pp. 798-810.

SANCHIS-GOMAR, F., PEREZ, L.M., JOYNE, M.J., LÖLLGEN, H., LUCIA, A. 2016. Endurance Exercise and the Heart: Friend or Foe? Sports Med, vol. 46, no 4, pp 459-66.

SHIROMA, E.J., SESSO, H.D., MOORTHY, M.V., BURING, J.E., LEE, I.M.: Do moderate -intensity and vigorous intensity physical activities reduce mortality rates to the same extent. J Am Heart Ass 2014; vol. 3, eooo802. 
SWEDISH NATIONAL INSTITUTE OF PUBLIC HEALTH. 2010. Physical activity in the prevention and treatment of disease. Professional Associations for physical activity. Stockholm, Sweden, http://www.fyss.se

VINA. J., SANCHIS-GOMAR, F., MARETINEZ-BELLO, V., GOMEZ-CABRERA, M.C., 2012. Exercise acts as a drug; the pharmacological benefits of exercise. Br J Pharmacol, vol. 167, no. 1, pp. 1-12.

ZUPET, P., LÖLLGEN, H., DEBRUYNE, A., BACHL, N., CUMMISKEY, J.., 2015. Exercise prescription for health Training recommendations. www. Efsma-scientific.com. 